\title{
Influencia del pH en la estabilidad de emulsiones elaboradas con proteínas de salvado de arroz
}

\author{
Maldonado, L. ${ }^{(1) *}$, Latorre, K. ${ }^{(1)}$, Rocha, P. ${ }^{(1)}$, Medrano, A. ${ }^{(1)}$, Abirached, C. ${ }^{(1)}$, Panizzolo, L. A. ${ }^{(1)}$ \\ (1) Departamento de Ciencia y Tecnología de Alimentos. Facultad de Química. Universidad de la República, Uruguay. \\ Contacto: lauramaldonado@fq.edu.uy
}

Recibido: 30/06/2011 - Aprobado: 17/10/2011

\begin{abstract}
$\underline{\text { Resumen }}$
Si bien las proteínas de origen animal en muchas instancias pueden tener mejores características funcionales que las proteínas de origen vegetal, el incremento de su costo puede favorecer al uso expansivo de las fitoproteínas como reemplazo. Una de las fuentes de proteínas de origen vegetal es el salvado de arroz, que se obtiene como subproducto en el proceso de pulido del arroz integral (Oryza santiva L) para producir el arroz blanco. Se estudió los procesos de cremado, floculación y coalescencia de emulsiones preparadas con proteínas del salvado de arroz a pH 6,0 y 8,0. La obtención de las proteínas del salvado de arroz se realizó en un medio alcalino, partiendo de salvado de arroz desengrasado. El proceso de desestabilización de las emulsiones se analizó a partir de los datos obtenidos por el método de retrodispersión de luz mediante un equipo Turbiscan 2000; en el caso del cremado los datos fueron ajustados a una cinética bifásica con una componente de segundo orden (hiperbólica) y otra con un comportamiento sigmoidal. Las emulsiones preparadas a pH 8 presentaron una mayor estabilidad frente al cremado, mientras que los procesos de floculación y coalescencia no fueron influenciados por los distintos valores de pH.

Palabras clave: Cinética, desestabilización, cremado, floculación, coalescencia.
\end{abstract}

\begin{abstract}
$\underline{\text { Abstract }}$
While animal protein in many instances may have better functional characteristics than vegetable proteins, their increasing cost may favour the expansive use of plant proteins as a replacement. One of the sources of plant protein is rice bran, which is obtained as a byproduct in the polishing process of integral rice (Oryza Santivan $L$ ) to produce white rice. The processes of creaming, flocculation and coalescence of emulsions prepared with rice bran protein at $\mathrm{pH} 6,0$ and 8,0, were studied. The obtaining of proteins from defatted rice bran was carried out in an alkaline environment. The process of destabilization of the emulsions was analyzed from data obtained by the method of backscattering of light by a Turbiscan 2000 equipment, in the case of creaming process the data fitted to a biphasic kinetics with a second order component (hyperbolic) and the other component with a sigmoidal behaviour. Emulsions prepared at $\mathrm{pH} 8$ showed a higher stability against creaming while flocculation and coalescence processes were not influenced by different $\mathrm{pH}$ values.

Keywords: Kinetics, Destabilization, Creaming, Size particle, Flocculation, Coalescence.
\end{abstract}

\section{Introducción}

La demanda de fuentes proteicas relativamente baratas que se puedan incorporar a los productos alimenticios incrementando así su valor agregado va mundialmente en aumento. Mucha de la reciente investigación en el tema ha estado dirigida al estudio de diferentes fuentes de fitoproteínas que puedan contribuir al aumento del valor nutricional de los productos alimenticios a un bajo costo (Gorinstein et al., 2002; Rangel et al., 2003; Sogi et al., 2002; Tomotake et al., 2002).

El salvado de arroz es una muy buena fuente alimentaria con un 13 $\%$ de proteína de buena calidad biológica, un alto contenido de fibras (20\%) y 12-15\% de lípidos, un perfil de ácidos grasos balanceado y un importante aporte de antioxidantes (Carroll, 1990). Uno de los principales aprovechamientos del salvado de arroz es la obtención de aceite. Este proceso es denominado desgrasado, por el cual se le extraen sus componentes lipídicos mediante extracción con solvente y posterior refinado. Debido a la presencia de enzimas como la lipasa y la lipooxigenasa es necesario, previo a la extracción de aceite, la inactivación de ellas por medio de un tratamiento térmico (Tang et al., 2002). La harina residual de la extracción del aceite del salvado así tratado constituye también un producto de gran valor nutritivo. El salvado de arroz contiene $12-20 \%$ de proteína, de buen valor biológico y propiedades nutracéuticas (Saunders, 1990). La proteína del salvado de arroz es de alta calidad (Juliano, 1985), el perfil de aminoácidos de la proteína del arroz es mejor que el de la caseína y el aislado proteico de soja, considerando los requerimientos de aminoácidos de niños de 2-5 años (Wang et al., 1999). Por otra parte, presenta baja alergenicidad, por lo que podría ser muy útil en formulaciones infantiles (Burks y Helm, 1994; Helm, 1996). Asimismo, la proteína del salvado de arroz ha trascendido por tener actividad anticancerígena (Kawamura y Muramoto, 1993).

La tendencia al consumo de productos naturales ha alcanzado ingredientes menores como los emulsificantes usados en la elaboración de emulsiones alimentarias. Desafortunadamente, y a excepción de la lecitina vegetal y animal, la mayoría de los emulsificantes usados en la industria alimentaria son sintéticos, por ejemplo, Tweens, Spans, monoglicéridos, etcetéra, a pesar de los esfuerzos realizados para encontrar alternativas a éstos (Yun et al., 2007).

El objetivo de este trabajo fue determinar la influencia del $\mathrm{pH}$ en las propiedades emulsificantes de las proteínas del salvado de arroz.

\section{Materiales y Métodos}

\section{Obtención del concentrado proteico de salvado de arroz}

Se partió de salvado de arroz (SA) desengrasado. Se determinó el contenido de proteínas en el SA por método de Kjeldahl. El concentrado proteico de salvado de arroz se obtuvo por solubilización 
de las proteínas en medio alcalino, $\mathrm{pH}$ 12,0, el pH se ajustó con $\mathrm{NaOH}$ $2 \mathrm{~N}$, la relación salvado:solvente fue 1:10, el tiempo de extracción 7 horas, luego se centrifugó a $10.000 \mathrm{rpm}, 20{ }^{\circ} \mathrm{C}, 30 \mathrm{~min}$. Sobre el sobrenadante se realizó precipitación isoeléctrica, llevando a pH 4,5 con $\mathrm{HCl} 6 \mathrm{~N}$, dejándolo a $4{ }^{\circ} \mathrm{C}$ durante la noche. Se separó el precipitado por centrifugación a $10.000 \mathrm{rpm}, 4{ }^{\circ} \mathrm{C}, 30 \mathrm{~min}$. Se resuspendió el precipitado en el mínimo volumen de agua destilada y se ajustó el pH a 12 con $\mathrm{NaOH} 2 \mathrm{~N}$. Se liofilizó 24 hs, según método de Chandi y Sogi (2007) modificado. Se determinó el contenido de proteína del concentrado obtenido por método de Lowry (1951).

\section{Solubilidad}

Se determinó la solubilidad del concentrado obtenido dispersándolo en solución de fosfato de sodio $10 \mathrm{mM}$ a pH 6,0 y 8,0 al $0,1 \% \mathrm{p} / \mathrm{v}$ durante 1 hora a temperatura ambiente y velocidad constante. Posteriormente, las muestras fueron centrifugadas a $10.000 \mathrm{~g}$ durante 10 minutos a $4{ }^{\circ} \mathrm{C}$. El contenido de proteína en el sobrenadante se determinó utilizando el método de Lowry (1951).

\section{Preparación de las emulsiones en estudio}

Se prepararon emulsiones aceite en agua, $\Phi=0,25(\mathrm{v} / \mathrm{v})$, con una concentración de proteína de $1,0 \mathrm{mg} / \mathrm{ml}$ en disolución de buffer fosfato $0,01 \mathrm{M}$, a pH 6,0 y 8,0, manteniendo constante la fuerza iónica en $0,03 \mathrm{M}$ con $\mathrm{NaCl}$. Las emulsiones fueron preparadas a temperatura ambiente con un homogenizador de alta velocidad Ultra-turrax T25 (IKA-Labortechnik, GmbH \& Co., Staufen, Alemania), utilizando el rotor S25 N-10 G a una velocidad de $20.000 \mathrm{rpm}$ durante un minuto a temperatura ambiente.

\section{Determinación de tamaño de partícula}

El análisis de tamaño de gota se realizó en emulsiones preparadas a $\mathrm{pH} 6,0$ y 8,0 , con y sin tratamiento con solución Tris/ $\mathrm{HCl} 0,05$ $\mathrm{M}, \mathrm{pH} 8,0$ SDS $1 \%$ en proporción 1:1, utilizando el analizador de tamaño de partículas Beckman Coulter LS 230. Beckman Coulter, Inc. Se determinó el diámetro promedio de gota $\mathrm{D}_{4,3}$. La diferencia de valores de diámetro promedio obtenidos en ausencia y presencia de la solución que contiene SDS se tomó como una medida de formación de flóculos o agregados en la emulsión (Anton et al., 2002). El grado de floculación se determinó según la ec. 1 .

$$
\left.\mathrm{GF}(\%)=\frac{\left(\mathrm{D}_{4,3}-\mathrm{D}_{4,3, \mathrm{sDS}}\right)}{\mathrm{D}_{4,3, \mathrm{sDS}}} \times 100 \quad \text { (ec. } 1\right)
$$

Donde: $\mathrm{D}_{43}$ : diámetro medio de Sauter para las emulsiones formadas; $\mathrm{D}_{4,3-\mathrm{SDS}}$ : $\mathrm{D}_{4,3}$ en presencia de SDS; GF: grado de floculación.

\section{Análisis de estabilidad de emulsiones aceite-agua}

La estabilidad global de las emulsiones se analizó con un analizador óptico vertical (Turbiscan Classic MA2000, Formulaction, Toulouse, France). Se registró la retrodispersión (RD \%) en función de la altura en la celda cada un minuto durante un período de 60 minutos y una única medida a las 24 horas. Los datos se tomaron a una altura de tubo de $10 \mathrm{~mm}$ desde la base. La cinética de desestabilización por cremado se estudió mediante los parámetros k y RD que surgen de la aplicación del modelo cinético propuesto por Panizzolo (2005), ec. 2.

$$
R D(t)=\left(\frac{R D_{h}}{R D_{h} K_{h} t+1}\right)+\left(\frac{2^{2} \cdot R D_{s}}{2^{2}+\left(k_{s} R D_{s}\right)^{2} \cdot t^{2}}\right)
$$

Donde: $\mathrm{RD}(\mathrm{t})$ : valor de $\mathrm{RD}$ a tiempo $\mathrm{t}$ a una altura de $10 \mathrm{~mm}$ desde la base del tubo; $\mathrm{k}_{\mathrm{h}} \mathrm{y}_{\mathrm{s}}$ : constantes de velocidad de variación de RD en función del tiempo para una cinética hiperbólica y sigmoidea, correspondientemente; $\mathrm{RD}_{\mathrm{h}}$ y $\mathrm{RD}_{\mathrm{s}}$ : parámetros de amplitud correspondientes a cinéticas hiperbólica y sigmoidea, respectivamente, tal que $\mathrm{RD}_{\mathrm{t}}+\mathrm{RD}=\mathrm{RDi}$; $\mathrm{RDi}$ es el valor de $\mathrm{RD}$ a $\mathrm{t}=0$. Los valores de

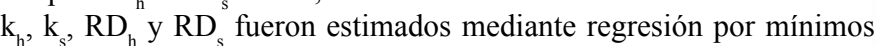
cuadrados.

La desestabilización global de las emulsiones por floculación y coalescencia en la fase crema se estudió en un periodo de almacenamiento de 24 hs mediante el cambio de los valores promedio de retrodispersión en el intervalo de altura de celda 54-56 mm (RDp5456) en función del tiempo. Se calculó el grado de desestabilización global según la ec. 3 (Palazolo et al., 2005).

$$
\mathrm{D}(\%)=\frac{\left(R D_{\mathrm{pmax}}-\mathrm{RD}_{\mathrm{p} 24}\right)}{R D_{\mathrm{pmax}}} \times 100
$$

Donde $\mathrm{D}(\%)$ : grado de desestabilización global; RDpmax: promedio del máximo valor de retrodispersión en el tiempo en el intervalo 54-56 mm, y RDp24: promedio del valor de retrodispersión a las 24 horas en el intervalo $54-56 \mathrm{~mm}$.

\section{Isoelectroenfoque}

Se realizó la determinación del pI de las proteínas del concentrado proteico por el método de Isoelectroenfoque. El concentrado de SA se dispersó en agua destilada, $1 \mathrm{mg} / \mathrm{ml}$. Se dializó la dispersión durante la noche y se procedió a la determinación usando un equipo PhastSystem (Pharmacia, Uppsala, Suecia). El pI de las muestras se determinó usando un kit de calibración en el rango de pH 3-9 (PhastGel IEF 3-9). El patrón fue reconstituido en $35 \mu \mathrm{L}$ de agua destilada. Se realizó tinción del gel con plata de acuerdo a las especificaciones del fabricante.

\section{Capacidad emulsionante}

Se evaluó la capacidad emulsionante (CE), cantidad de aceite que puede emulsionar una solución de proteína a una concentración dada, al momento en que se produce la inversión de la emulsión (Wagner, 2000) por método conductimétrico. Se agregó aceite a una solución de proteína de $1,0 \mathrm{mg} / \mathrm{ml}$ en disolución de fosfato de sodio $0,01 \mathrm{M}$, a $\mathrm{pH}$ 6,0 y 8,0 , fuerza iónica 0,03 . Se agitó luego de cada agregado con un homogenizador Ultra-turrax T25 (IKA-Labortechnik, GmbH \& Co., Staufen, Alemania), utilizando el rotor S25 N-10G a una velocidad de $20.000 \mathrm{rpm}$ durante un minuto, y se registró la conductividad (conductímetro Oakton). La inversión se determinó como el volumen de aceite agregado al cual la conductividad disminuye a valores cercanos a cero. Se calculó $\mathrm{CE}(\%)=($ Volumen aceite/ Volumen total) $* 100$

\section{Análisis estadístico}

El tratamiento estadístico de datos se realizó en todos los casos mediante análisis de varianza (ANOVA) con $\alpha=0,05$ y la comparación de medias por la prueba de mínimas diferencias significativas (LSD) $\operatorname{con} \alpha=0,05$.

\section{Resultados y Discusión}

El contenido de proteínas del SA desgrasado por método de Kjeldahl fue de 40,2 \%. El mayor rendimiento de extracción se obtuvo a pH 12,0 en una relación salvado:solvente de 1:10 durante un tiempo de extracción de 7 horas, posterior precipitación isoeléctrica a pH 4,5.

El contenido proteico del concentrado fue $66.9 \pm 2.9 \%$, por lo que el rendimiento de extracción resultó en $10.14 \pm 0.59 \%$, lo cual 
es bajo comparado con el informado por otros autores (Connor et al., 1976), quienes obtuvieron concentrados de hasta el $82 \%$. El rendimiento de extracción pudo verse afectado por el tratamiento térmico recibido por el SA. Cabe destacar que un mayor contenido proteico en el concentrado obtenido respecto al salvado de arroz se debe a la forma de extracción de las proteínas. Luego de colocar el SA en un medio alcalino en el cual se solubilizan las proteínas, se liofilizó el extracto acuoso y se obtuvo un polvo amarillento, con un contenido de proteínas del $66.9 \%$, siendo ésta una forma de concentración de las mismas.

La solubilidad del concentrado fue $19,8 \pm 0,2 \%$ y $60 \pm 1 \%$ a pH 6,0 y 8,0 , respectivamente. El resultado es significativamente mayor a $\mathrm{pH} 8,0$.

En la Figura 1 se muestran las curvas de distribución de tamaño de partícula a pH 6,0 y 8,0. Tanto a pH 6,0 como 8,0 se observó una distribución bimodal, correspondiente a dos poblaciones de partículas claramente definidas. Los valores obtenidos de $\mathrm{D}_{4,3}$ para $\mathrm{pH} 6,0$ y 8,0 fueron $62,7 \pm 0,6 \mu \mathrm{m}$ y $36,9 \pm 1,9 \mu \mathrm{m}$, respectivamente, lo cual fue significativamente menor a $\mathrm{pH} 8,0$.

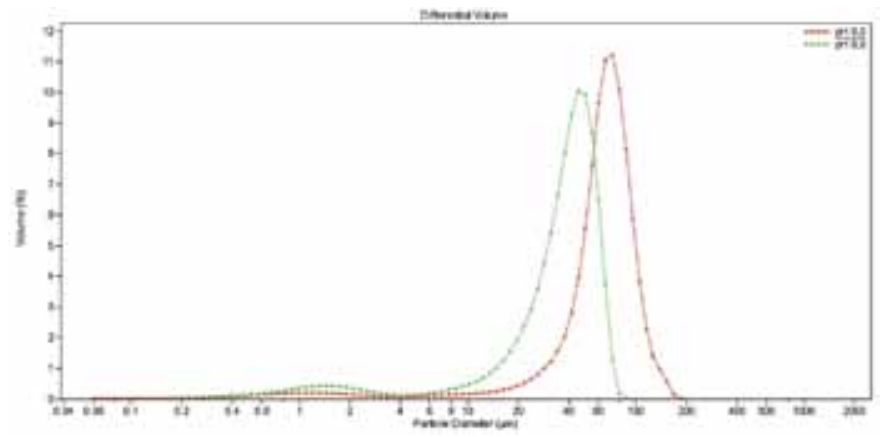

Figura 1. Distribución de tamaño de partícula para emulsiones formadas a pH 6,0 y 8,0 (Volumen vs Diámetro de partícula).

El análisis de diámetro de partícula con SDS reveló que la desestabilización inicial por floculación y coalescencia no fue apreciable.

En la Figura 2 se muestran los perfiles de RD en función de la altura del tubo, que surgen del estudio de estabilidad de las emulsiones formadas. En la zona inferior del tubo de muestra, zona I (altura $10 \mathrm{~mm}$ ), se observó una disminución de los valores de RD con el transcurso de tiempo, lo que evidencia el desarrollo de un proceso de cremado, mientras que en la zona II RD aumentó, producto de la acumulación gotas de aceite en la parte superior del tubo (fase crema).

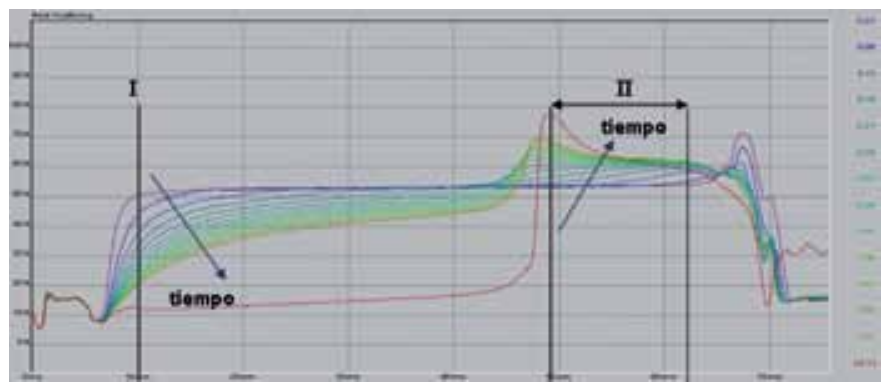

Figura 2. Perfil de retrodispersión (RD\%) en función de la altura de la celda $(\mathrm{mm})$ para una emulsión formada a $\mathrm{pH} 8,0$. Las flechas indican el sentido de aumento del tiempo. I: zona inferior del tubo (altura $10 \mathrm{~mm}$ ), II: zona superior del tubo en la que se observa la fase crema

Las curvas de variación de los valores promedio de RD (RDp) a una altura de $10 \mathrm{~mm}$, para emulsiones formadas a $\mathrm{pH} 6,0$ y 8,0 se muestran en la Figura 3. Es posible constatar que las curvas presentan un perfil sigmoideo.

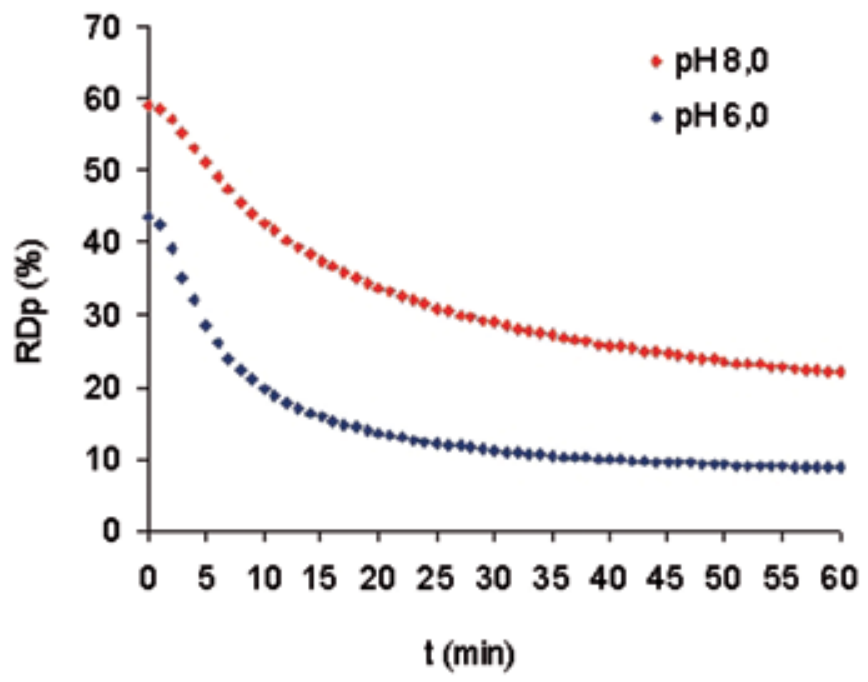

Figura 3. Curvas de $\mathrm{RD}_{\mathrm{p}}$ versus tiempo para emulsiones formadas con el concentrado proteico de SA a pH 6,0 ( $\diamond$ y 8,0 ( $\diamond$ ). El análisis de datos se realizó a $10 \mathrm{~mm}$ desde la base del tubo.

Según Panizzolo (2005), las emulsiones que presentan un perfil de $\mathrm{RD}_{\mathrm{p}}$ de forma sigmoidea, se describen adecuadamente mediante la ec. 2. El modelo cinético describe la desestabilización por cremado e implica la existencia de dos poblaciones de partículas. El término sigmoideo se vincula a las partículas de mayor tamaño, mientras que el hiperbólico se asocia a las partículas más pequeñas. En la Figura 4 se muestra la contribución de cada término de la ec. 2 a la cinética de desestabilización. Allí se evidencia que esta es la suma de una cinética hiperbólica y otra sigmoidea. El término sigmoideo contribuye sólo al principio, mientras que a tiempos mayores a $20 \mathrm{~min}$ es el término hiperbólico el que más contribuye a la cinética de desestabilización.

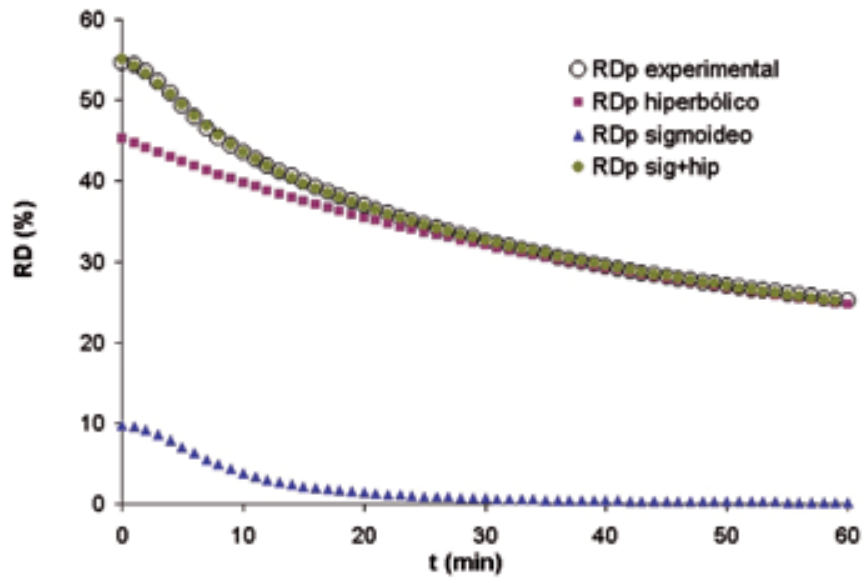

Figura 4. (o) Valores de RDp experimentales en función de tiempo para una emulsión formada a $\mathrm{pH} 8,0$. (口) Variación de RDp ajustando los datos a una cinética hiperbólica. (A) Variación de RDp ajustando los datos a una cinética sigmoidea. (c) Valores de RDp para la suma de las cinéticas hiperbólica y sigmoidea (sig+hip), ec. 2.

En la Tabla 1 se muestran los valores obtenidos para los parámetros $\mathrm{k}_{\mathrm{s}} \mathrm{y} \mathrm{k}_{\mathrm{h}}$ de la ec. 2 y los valores de $\mathrm{RD}_{\mathrm{i}}$. Se puede observar que $\mathrm{RD}_{\mathrm{i}}$ a $\mathrm{pH}$ 8 es considerablemente mayor; los valores de $\mathrm{RD}_{\mathrm{i}}$ están vinculados con $\phi$ y el tamaño medio de las gotas de la emulsión en función de la altura del dispositivo de medida (Abismaïl et al., 2000), por tanto implica que a pH 8 el tamaño de gota sería mucho menor. Este resultado se correlaciona muy bien con el obtenido en las curvas de distribución de tamaño de partícula. A pH 6,0 los valores de $\mathrm{k}_{\mathrm{h}}$ fueron mayores, mientras que no existieron diferencias significativas en los valores de $\mathrm{k}_{\mathrm{s}}$, lo que implica una desestabilización más rápida a este $\mathrm{pH}$. 


\begin{tabular}{|c|c|c|}
\hline PROMEDIOS & pH 8,00 & pH 6,00 \\
\hline $\mathrm{k}_{\mathrm{h}} \times 10^{4}\left(\mathrm{~min}^{-1}\right)$ & $5 \pm 1^{\mathrm{a}}$ & $9 \pm 1^{\mathrm{b}}$ \\
\hline $\mathrm{k}_{\mathrm{s}} \times 10^{2}\left(\mathrm{~min}^{-2}\right)$ & $1,6 \pm 0,3^{\mathrm{a}}$ & $1,3 \pm 0,4^{\mathrm{a}}$ \\
\hline $\mathrm{RD}_{\mathrm{i}}(\%)$ & $55 \pm 3^{\mathrm{a}}$ & $46 \pm 2^{\mathrm{b}}$ \\
\hline
\end{tabular}

Tabla 1. Parámetros cinéticos de desestabilización de emulsiones formadas con proteínas de $S A$ a pH 6,0 y $8,0 . R D_{\text {i }}=R D_{s}+R D_{h}$ a tiempo 0 . Letras diferentes en la misma fila significa qúe existen diferencias significativas entre las muestras con un $\alpha \leq 0,5$.

La desestabilización por floculación o coalescencia en la fase crema fue despreciable durante las 24 hs de almacenamiento de las emulsiones. Los valores de D (\%) fueron $0,7 \pm 1,3 \%$ y $1.0 \pm 1.5 \%$ a pH 6,0 y 8,0 , respectivamente.

Por método de isoelectroenfoque se observaron bandas a $\mathrm{pH} 6,4$ (imagen no mostrada) correspondientes al punto isoeléctrico de una o más proteínas del concentrado, indicando un mayor grado de agregación a este $\mathrm{pH}$.

La capacidad emulsionante del concentrado fue de $66 \pm 1 \%$ y 64 $\pm 2 \%$ para $\mathrm{pH} 8,0$ y 6,0 , respectivamente, sin que existan diferencias significativas entre ambos $\mathrm{pH}$.

Con el fin de proveer buenas propiedades emulsificantes, las proteínas usadas como ingredientes en alimentos deben poseer una alta solubilidad. La solubilidad obtenida a $\mathrm{pH}$ 8,0 explica la mayor estabilidad de las emulsiones a este $\mathrm{pH}$, ya que la proteína debe estar soluble para migrar a la interfase y ubicarse en ella. La solubilidad se ve modificada por varios factores como el $\mathrm{pH}$, la fuerza iónica, temperatura, etcétera. La variación de alguna de estas condiciones promueve cambios en las interacciones electrostáticas e hidrofóbicas en la estructura de las proteínas, llevando a diferentes conformaciones, lo cual afecta las propiedades funcionales. Un aumento en las interacciones hidrofóbicas resulta en una tendencia de las proteínas a formar agregados insolubles, disminuyendo por tanto la solubilidad, lo que sucede en rangos de $\mathrm{pH}$ cercanos al $\mathrm{pI}$.

\section{Conclusiones}

El análisis de los resultados obtenidos permite concluir que el concentrado de salvado de arroz es un mejor agente emulsificante a $\mathrm{pH}$ 8,0 . Las emulsiones presentan menor tamaño de partícula y por tanto mayor estabilidad, ligado a una mayor solubilidad del concentrado a este $\mathrm{pH}$. El mecanismo de desestabilización que primó fue el cremado. No existieron diferencias significativas entre $\mathrm{pH}$ 6,0 y 8,0 en cuanto a la capacidad emulsionante.

\section{Referencias}

- ABISMAÏL, B.; CANSELIER, J. P.; WILHELM, A.M.; DELMAS, H. Y GOURDON C. Emulsification processes: on-line study by multiple light scattering measurements. En: Ultrasonics Sonochemistry. 2000, (7):187-192.

- ANTON, M.; BEAUMAL, V.; BROSSARD, C.; LLAMAS, G.; LE DENMAT, M. Droplet flocculation and physical stability of oil-in-water emulsions prepared with hen egg yolk. En: ANTON, M. Food emulsions and dispersions. Kerala: Research Signpost, 2002. pp.15-28.

- BURKS, A. W.; HELM, R. M. Hypoallerginicity of rice protein. En: AACC. The annual meeting of the American Association of Cereal Chemists, (Nashville 23-27 de octubre de 1994). Nashville: AACC, 1994.

- CARROLL, L. Functional properties and applications of stabilized rice bran in bakery products. En: Food Technology. 1990, 44 (4):74-76.

- CHANDI, G. K.; SOGI, D. S. Functional properties of rice bran protein concentrates. En: Journal of Food Engineering. 2007, 79:592-597
- CONNOR, M.A.; SAUNDERS, R.M.; KOHLER, G.O. Rice bran protein concentrates obtained by wet alkaline extraction. En: Cereal Chemistry. 1976, 53(4):488.

- GORINSTEIN, S.; PAWELZLK, E.; LICON, E. D.; HARUENKIT, R.; WEISZ, M.; TRAKHTENBERG, S. Characterisation of pseudocereal and cereal proteins by protein and amino acid analyses. En: Journal of the Science of Food and Agriculture. 2002, 82:886-891.

- HELM, R. M.; BURKS, A. W. Hypoallergenicity of rice bran protein. En: Cereal Foods World. 1996, 41 (11):839-843.

- JULIANO, B. O. Production and utilization of rice. En: JULIANO, B. O. Rice: Chemistry and Technology. 2a ed. Saint Paul: Amer Assn of Cereal Chemists, 1985. pp. 1-16

- KAWAMURA, Y.; MURAMOTO, M. Antitumorigenic and immunoactive protein and peptide factors in foodstuff. 2. Antitumorigenic factors in rice bran. En: WALDRON, K. W.; JOHNSON, I. T. FENWICK, G.R. Food and cancer prevention. Chemical and biological Aspects. Philadelphia: Woodhead, 1993. pp. 331-401

- LOWRY, O. H.; ROSEBROUGH, N. J.; FARR, A. L.; RANDALL, R. J. Protein measurement with the folin-phenol reagent. En: Journal of Biological Chemistry . 1951, 193:265-275.

- PALAZOLO, G.; SORGENTINI, D.; WAGNER, J. R. Coalescence and flocculation in $\mathrm{o} / \mathrm{w}$ emulsions of native and denatured whey soy proteins in comparison with soy protein isolates. En: Food Hydrocolloids. 2005, 19:595-604.

- PANIZZOLO, L. A. Modificación de proteinas por vía enzimática. Análisis de la relación estructura-funcionalidad de los productos de hidrólisis. Montevideo: Facultad de Química de la Universidad de la República, 2005. (Tesis Doctoral).

- RANGEL, A.; DOMONT, G. B.; PEDROSA, C. Y FERRIERA, S. T. Functional properties of purified vicilins from cowpea (Vigna unguiculata) and Pea (Pisum sativum) and cowpea protein isolate. En: Journal of Agricultural and Food Chemistry. 2003, 51:57925797.

- SAUNDERS, R. M. The properties of rice bran as a food stuff. En: Cereal Foods World. 1990, 35:632-662.

- SOGI, D. S.; GARG, S. K.; BAWA, A. S. Functional properties of seed meals and protein concentrates from tomato processing waste. En: Journal of Food Science. 2002, 67:2997-3001.

- TANG, S.; HETTIARACHCHY, N. S.; Y SHELLHAMMER, T. H. Protein Extraction from Heat-Stabilized Defatted Rice Bran. 1. Physical Processing and Enzyme Treatments. En: Journal of Agricultural and Food Chemistry. 2002, 50:7444-7448.

- TOMOTAKE, H.; SHIMAOKA, I.; KAYASHITA, J.; NAKAJOH, M. Y KATO, N. Physicochemical and functional properties of buckwheat protein product. En: Journal of Agricultural and Food Chemistry. 2002, 50:2125-2129.

- WAGNER, J. R. Propiedades superficiales. En: PILOSOF, A. M. R.; BARTHOLOMAI, G. Caracterización funcional y estructural de proteinas. Buenos Aires: Eudeba, 2000. pp. 41-70.

- WANG, M.; HETTIARCHCHY, N. S.; QI, M.; BURKS, W.; SIEBENMORGEN, T. Preparation and functional properties of rice bran protein isolate. En: Journal of Agricultural and Food Chemistry. 1999, 47: 411-416.

- YUN, S. E.; TONG, S.T. Isolation and investigation of emulsifying properties of surface-active substances from rice bran. En: Food Hydrocolloids. 2007, 21(2):838-843 